\title{
Radial Electron Momentum Densities of Colloidal CdSe Nanocrystals Determined by Positron Beam Analysis
}

\author{
A.B. Denison, R. Meulenberg, S.W.H. Eijt, A. Van Veen, \\ P.E. Mijnarends, B. Barbiellini, A. Bansil, C. Fischer, \\ M.H. Weber, K.G. Lynn
}

This article was submitted to

$13^{\text {th }}$ International Conference on Positron Annihilation, ICPA-13 Kyoto, Japan

September 7-13, 2003

U.S. Department of Energy

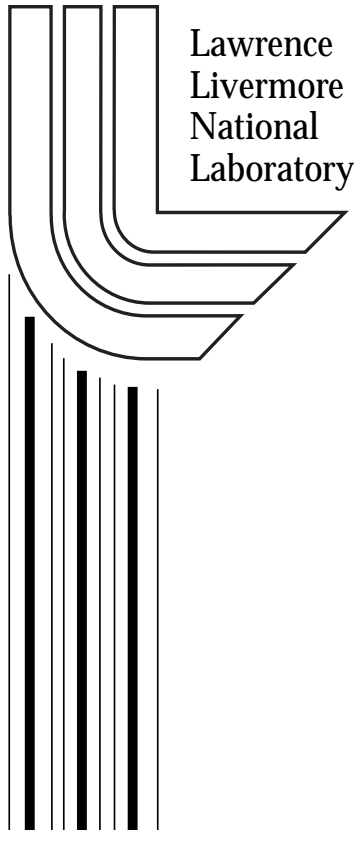

\section{July 31, 2003}




\section{DISCLAIMER}

This document was prepared as an account of work sponsored by an agency of the United States Government. Neither the United States Government nor the University of California nor any of their employees, makes any warranty, express or implied, or assumes any legal liability or responsibility for the accuracy, completeness, or usefulness of any information, apparatus, product, or process disclosed, or represents that its use would not infringe privately owned rights. Reference herein to any specific commercial product, process, or service by trade name, trademark, manufacturer, or otherwise, does not necessarily constitute or imply its endorsement, recommendation, or favoring by the United States Government or the University of California. The views and opinions of authors expressed herein do not necessarily state or reflect those of the United States Government or the University of California, and shall not be used for advertising or product endorsement purposes.

This is a preprint of a paper intended for publication in a journal or proceedings. Since changes may be made before publication, this preprint is made available with the understanding that it will not be cited or reproduced without the permission of the author.

This report has been reproduced directly from the best available copy.

Available electronically at http://www.doc.gov/bridge

Available for a processing fee to U.S. Department of Energy

And its contractors in paper from

U.S. Department of Energy

Office of Scientific and Technical Information

P.O. Box 62

Oak Ridge, TN 37831-0062

Telephone: (865) 576-8401

Facsimile: (865) 576-5728

E-mail: reports@adonis.osti.gov

Available for the sale to the public from

U.S. Department of Commerce

National Technical Information Service

5285 Port Royal Road

Springfield, VA 22161

Telephone: (800) 553-6847

Facsimile: (703) 605-6900

E-mail: orders@ntis.fedworld.gov

Online ordering: http://www.ntis.gov/ordering.htm

OR

Lawrence Livermore National Laboratory

Technical Information Department's Digital Library

http://www.llnl.gov/tid/Library.html 


\title{
Radial Electron Momentum Densities of Colloidal CdSe Nanocrystals Determined by Positron Beam Analysis
}

\author{
A. B. Denison ${ }^{1}$, R. Meulenberg ${ }^{1}$, S. W. H. Eijt ${ }^{2}$, A. Van Veen ${ }^{2}$, P. E. \\ Mijnarends ${ }^{2,3}$, B. Barbiellini ${ }^{3}$, A. Bansil ${ }^{3}$, C. Fischer ${ }^{4}$, M.H. Weber ${ }^{4}$, K.G. Lynn ${ }^{4}$ \\ ${ }^{1}$ Lawrence Livermore National Laboratory, 7000 East Avenue, Livermore, CA 94551, USA \\ ${ }^{2}$ Interfaculty Reactor Institute, Delft University of Technology, Delft, The Netherlands \\ ${ }^{3}$ Northeastern University, Department of Physics, Boston, MA 02115, USA \\ ${ }^{4}$ Washington State University, Center for Materials Research, Pullman, WA 99164, USA
}

Keywords: positron annihilation, CdSe, quantum dots, electronic structure, surface

\begin{abstract}
We present depth-resolved positron 2D angular correlation of annihilation radiation (2DACAR) experiments on CdSe quantum dots in the diameter range from 2.5 to $6 \mathrm{~nm}$, deposited as micrometer thin layers. The average radial distribution of the valence electron momentum density (EMD) of CdSe quantum dots has been extracted, which reveals a systematic dependence upon particle size. The quantum confinement related changes and their size scaling observable at the Jones zone momentum of $\sim 0.8$ a.u. seem to agree with the previous coincidence Doppler study. In addition, the average radial EMD shows an increase in the low-momentum range $(<0.6$ a.u. $)$ and a reduction in the high-momentum range ( $>1.6$ a.u.) with respect to that measured on a bulk CdSe single crystal. Possible origins of these are described. First-principles calculations based on the Korringa-KohnRostoker (KKR) method were performed to gain a better insight.
\end{abstract}

\section{Introduction}

As the need for nano-structures for technological applications grows, the ability to characterize the electronic properties and integrity of the structure itself becomes of paramount importance. Positron spectroscopy is well-known to be one of the most sensitive probes of electron momentum density and defects in bulk materials, surfaces, and interfaces. We have continued measurements on CdSe quantum dots (QD) using the facilities at the Delft University of Technology (DUT) - Interfaculty Reactor Institute. High-statistics runs using 2D-ACAR have confirmed earlier results [1] at Washington State University (WSU) and show that the effects of quantum confinement follow an inverse square law $1 / d^{2}$ upon particle diameter $d$ [1], in contrast to the $1 / d$ law expected from firstprinciples calculations on the confined Homogeneous Electron Gas (HEG) [2]. In addition, the data indicate that the changes observed in the low- and high-momentum regions for the quantum dots in comparison with bulk annihilation spectra can be systematically explored.

\section{Experimental}

The samples were prepared in the same manner as for the earlier measurements [1] by the colloidal method [3] resulting in the surfactant coating, nominally TOPO (tri-octyl phosphine oxide) used to terminate the growth process in solution. Samples with dots of average diameter $d$ of $2.5 \mathrm{~nm}, 2.9$ $\mathrm{nm}, 3.6 \mathrm{~nm}$ and an agglomeration with an average diameter of $\sim 6 \mathrm{~nm}$ were subsequently measured at $8 \mathrm{keV}$ positron implantation energy and compared with a bulk single crystal with the hexagonal [0001] axis perpendicular to the sample surface. The randomly oriented CdSe quantum dot samples were deposited on $\mathrm{Si}(100)$ substrates to a thickness beyond the depth range of positrons at $25 \mathrm{keV}$ $(\sim 3 \mu \mathrm{m})$, as shown by Doppler depth-profiling. 2D-ACAR distributions at room temperature were obtained with the POSH-ACAR facility [4] at $8 \mathrm{keV}$ positron implantation energy at an overall 
resolution of $1.4 \times 1.4\left(10^{-3} m_{0} c\right)^{2}$. One 2D-ACAR distribution $N\left(p_{x}, p_{y}\right)$ was obtained of a highquality bulk CdSe single crystal with the [0001] axis along the $p_{x}$ direction, using a ${ }^{22} \mathrm{Na}$ source.

\section{Results}

The 2D-ACAR results on the quantum dot samples and the CdSe single crystal confirmed the absence of a p-Ps contribution [1]. Further, the anisotropy of the 2D-ACAR distributions of the QD samples is weak relative to that of the CdSe single crystal and shows only an irregular noise-related pattern, demonstrating that the quantum dot particles are randomly oriented and no substrate contribution is observed. Consequently, the average radial electron momentum distribution $\rho(p)=\langle\rho(|\mathbf{p}|)\rangle$ for the CdSe nanocrystals can be extracted (Figure 1). A similar procedure involving the average isotropic part of the 2D-ACAR distribution has been used to obtain $\rho(p)$ of bulk CdSe. A clear broadening above $\sim 1.0$ a.u. is visible, related to quantum confinement. A preliminary analysis seems to confirm that the broadening scales as $1 / d^{2}[1]$. Figure 2 shows the ratio $\Delta \rho / \rho_{\text {bulk }}$ of the difference between the average EMD for a QD with a diameter of $3.6 \mathrm{~nm}$ and bulk CdSe. Three regions can clearly be distinguished. Region I below $\sim 0.6$ a.u. shows the relative increase of $\rho_{\mathrm{QD}}(p)$ over that of bulk CdSe. Region II between $\sim 0.6$ a.u. and $\sim 1.6$ a.u. shows a bump peaking at $\sim 1.2$ a.u. The relative heights in this region increase as $1 / d^{2}$ with decreasing size as observed earlier [1]. In region III a marked decrease with a minimum occurring at $~ 2-2.5$ a.u. is observed.

\section{KKR calculations}

To gain a better insight in the EMD of CdSe, first-principle KKR calculations [6] were performed for bulk mono-crystalline CdSe. Details of the calculations will be presented elsewhere, but basically the KKR calculations were made using the wave functions calculated in the Independent Particle Model for zincblende-structure CdSe. The crystal potentials had the muffin-tin form. Although the structure of the CdSe quantum dots is wurtzite [8] the zincblende structure is expected to give a similar average radial electron momentum density. Figure 3 displays the calculated average radial momentum density of the relevant energy bands (see inset of Fig. 3) and the separate contributions from the valence bands $\mathrm{Se}(4 \mathrm{p}), \mathrm{Cd}(4 \mathrm{~d})$ and $\mathrm{Se}(4 \mathrm{~s})$. The overall radial average _( $p)$ calculated agrees rather well with the measured momentum density for the bulk (Figure 1). Therefore, the defect concentration of the single crystal sample is expected to be very low. These calculations indicate that in the low-p region (Region I) the Se $4 p$ and $4 s$ bands are the main contributors, whereas in the high-p region (Region III) it is the $\mathrm{Cd}(4 \mathrm{~d})$ band. The observed broadening in the Region II, near the Jones zone, is therefore mainly affected by quantum confinement of the valence electrons $(\operatorname{Se}(4 \mathrm{p}))$.

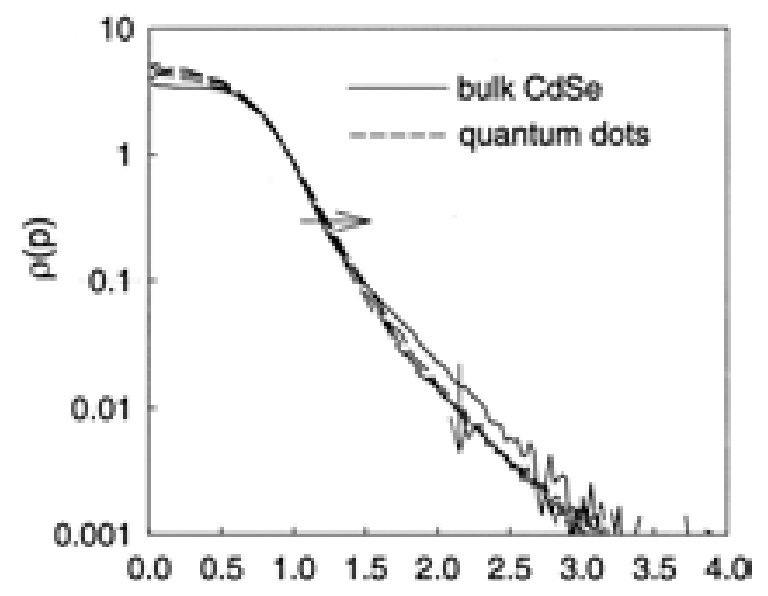

Fig. 1: Average radial EMD distribution $\rho(p)$ for the series of QD samples and for a CdSe single crystal. The arrows indicate the systematic broadening above the Jones zone momentum ( $~ 0.8$ a.u.) and the reduction in the high-momentum range.

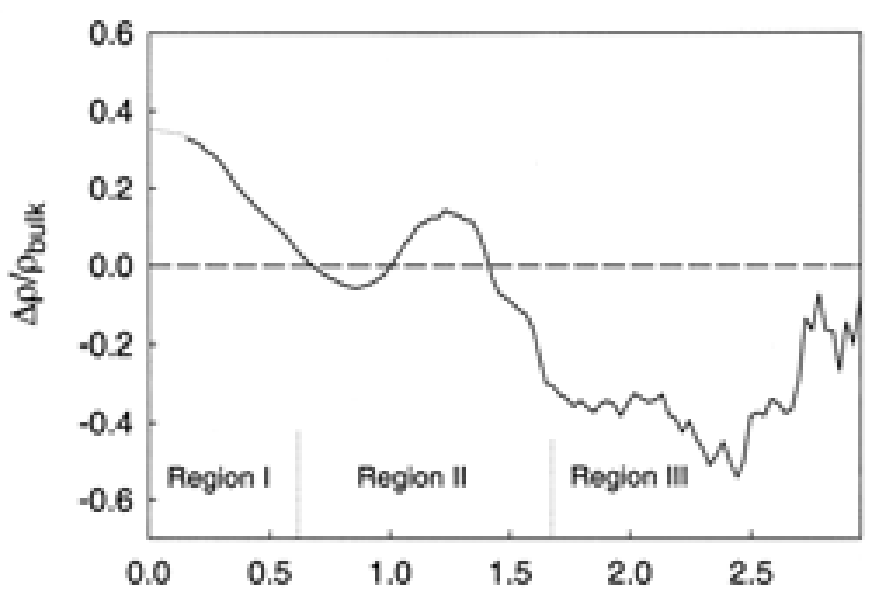

Fig. 2: Ratio curve $\Delta \rho / \rho_{\text {bulk }}$ between $\rho(p)$ of the $3.6 \mathrm{~nm}$ CdSe quantum dot sample and $\rho(p)$ of bulk CdSe, showing three different regions. 


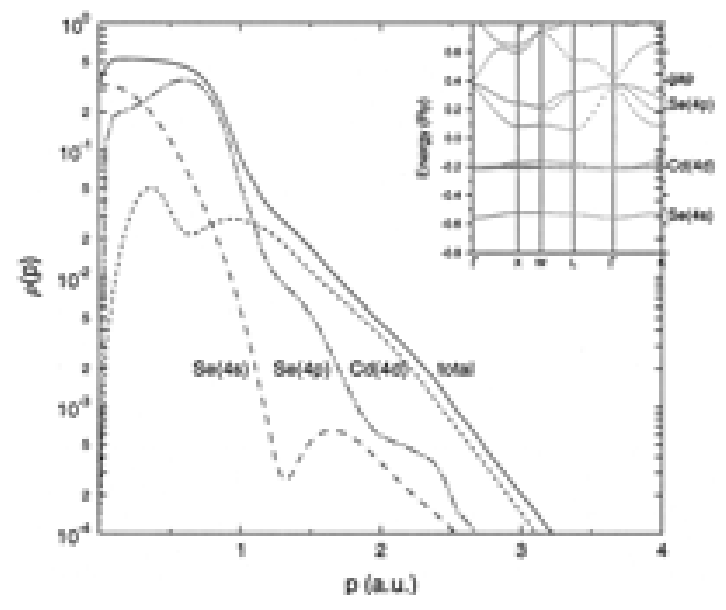

Fig. 3: Directionally averaged momentum density <_ $(|\mathbf{p}|)>$ obtained by first principles KKR-calculations on bulk mono-crystalline zincblende CdSe. The contributions from the occupied $\operatorname{Se}(4 \mathrm{p}), \operatorname{Se}(4 \mathrm{~s})$ and $\mathrm{Cd}(4 \mathrm{~d})$ electron bands are shown. The inset shows the band structure for bulk zincblende CdSe.

Discussion: The new high-statistics 2D-ACAR runs for radially averaged momentum spectra show a clear deviation of the quantum dot spectra for all sizes from the measured bulk spectrum. Speculating on its origin, at least four factors may in principle contribute to the changes observed in the momentum regions I and III. Firstly, the momentum density of the TOPO ligand molecules is not known. Secondly, the increase at low momenta and reduction at high momenta may be related to a weak localisation of the positron at $\mathrm{Cd}$ vacancies. However, the preparation procedure followed leads in general to high-purity quantum dot crystals. Thirdly, they may be related to the electron (and positron) spatial charge distributions in the quantum dots, the understanding of which requires abinitio calculations on quantum dot systems [7]. Fourthly, the fact that the surfactant coating is not present on the bulk crystal may contribute to the differences. It has been shown experimentally using ${ }^{31} \mathrm{P}$ NMR [5] that the preferred binding TOPO site is Cd. Referring to Figure 2 these results seem to be consistent with Region I showing a $\_/ \rho_{\text {bulk }}$ greater than zero, arising from excess $\operatorname{Se}(4 \mathrm{~s})$ and $\mathrm{Se}(4 \mathrm{p})$ momentum density. Region III on the other hand indicates a reduced $\mathrm{Cd}(4 \mathrm{~d})$ contribution. Probably the positron wave function near the surface annihilates preferentially with Se atoms whose orbitals are not tied up by bonding to the TOPO surfactant cap as are essentially $100 \%$ of the Cd surface atoms [5]. If this is the case, the positron annihilation technique could well be a powerful probe of the surface and interface of capped or core-shell quantum structures. Caution must be exercised, however, with respect to the uniqueness of this explanation. Further, the 2D-ACAR measurements on the bulk crystal have only been made for one single orientation. It is well-known that the momentum density is anisotropic [6]. In order to make a fair comparison between the capped nano-structures and the non-capped bulk it will be necessary to average measurements for other orientations or measure a distribution of large randomly oriented grains of uncapped CdSe. These experiments are presently in preparation. Additionally, samples of CdSe quantum dots are being capped with other capping agents. We are presently preparing samples with surfactant capping using amines and thiols where capping to Se is more probable. Results of these measurements will be presented elsewhere.

Acknowledgements This work was performed under the auspices of the U.S. Department of Energy at the University of California/Lawrence Livermore National Laboratory under Contract No. W-7405-Eng-48. and the Division of Materials Science Office of Basic Energy Sciendes. This computational work was

\section{References} sponsored by the Stichting NCF (NWO) for the use of supercomputer facilities.

[1] M.H. Weber, K. G. Lynn, B. Barbiellini, P. A. Sterne, A. B. Denison: Phys. Rev. B. 66 (2002), 041305

[2] R. Saniz, B. Barbiellini, A. B. Denison: Phys. Rev. B 65 (2002), 245310

[3] C.B. Murray, D. Norris, M.G. Bawendi: J. Am. Chem. Soc., 115 (1993), p. 8706

[4] C.V. Falub, S.W.H. Eijt, P.E. Mijnarends, H. Schut, A. van Veen: Nucl. Instr. \& Meth. Phys.

Res. A 488 (2002), p. 478

[5] L. Becerra, C.B. Murray, R. Griffen, M.G. Bawendi: J. Chem. Phys., 100 (1994), p. 3297

[6] P.E. Mijnarends and A. Bansil, in Positron Spectroscopy of Solids, edited by A.Dupasquier and A.P. Mills, Jr., (IOS, Amsterdam, 1995), p 25.

[7] A. Puzder, A.J. Williamson, J. C. Grossmann and G. Galli: Phys. Rev. Lett. 88 (2002), 097401

[8] X. Peng, M. Schlamp, A. Kadavinich, A.P. Alivisatos: J. Am. Chem. Soc., 119 (1997), p. 7019 